\title{
Superação da Profundidade de Semeadura e Densidades de PALHA PARA Mucuna aterrima, Mucuna deeringiana E Mucuna cinerea ${ }^{1}$
}

\author{
Overcoming Depth Sowing and Mulch Density in Mucuna aterrima, Mucuna deeringiana and \\ Mucuna cinerea
}

\author{
SILVA. G.B.F. ${ }^{2}$, AZANIA, C.A.M..$^{3}$, NOVO, M.C.S.S. ${ }^{4}$, WUTKE, E.B. ${ }^{5}$, ZERA, F.S. ${ }^{6}$ e \\ AZANIA, A.A.P.M. ${ }^{7}$
}

\begin{abstract}
RESUMO - O experimento foi desenvolvido em casa de vegetação, com o objetivo de verificar a capacidade das sementes de Mucuna aterrima, Mucuna cinerea e Mucuna deeringiana para superar diferentes profundidades do solo acrescido de densidades de palha de cana-deaçúcar. O delineamento utilizado foi o inteiramente casualizado; os tratamentos foram distribuidos em esquema fatorial $3 \times 3 \times 3$, com quatro repetições. Os fatores foram constituídos por camada de palha $(0,5$ e $10 \mathrm{~cm})$, espécies de Mucuna ( $M$. aterrima, $M$. cinerea e $M$. deeringiana) e profundidade de semeadura (1, 4 e $8 \mathrm{~cm})$. As espécies de Mucuna superaram até $23 \mathrm{t} \mathrm{ha}^{-1}$ de palha $(10 \mathrm{~cm})$ e até $8 \mathrm{~cm}$ de profundidade de semeadura, expondo as partes aéreas à superfície a partir dos 10 dias após emergência (DAS); elas foram capazes também de desenvolver a altura (>96 cm por planta), a área foliar $\left(>723 \mathrm{~cm}^{-2}\right.$ por planta) e o acúmulo de massa seca (>2,09 g por planta) até os 35 DAS.
\end{abstract}

Palavras-chave: cana-de-açúcar, palha, planta daninha.

\begin{abstract}
The experiment was conducted in a greenhouse to verify the ability of Mucuna aterrima, Mucuna cinerea and Mucuna deeringiana seeds to overcome different soil depths plus sugarcane straw densities. The experimental design was completely randomized with the treatments arranged in a factorial scheme $3 \times 3 \times 3$ and four replications. The factors consisted of straw layers (0, 5, and $10 \mathrm{~cm}$ ), Mucuna species (M. aterrima, $\boldsymbol{M}$. cinerea and $\boldsymbol{M}$. deeringiana) and sowing depth $\left(1,4\right.$ and $8 \mathrm{~cm}$ ). The Mucuna species exceeded the sowing depth by $23 t \mathrm{ha}^{-1}$ straw and up to $8 \mathrm{~cm}$, exposing the shoot to the surface from 10 days after emergence and also being able to develop height (> $96 \mathrm{~cm}$ per plant), leaf area (> $723 \mathrm{~cm}^{-2}$ per plant) and dry mass accumulation (> $2.09 \mathrm{~g}$ per plant) up to $35 \mathrm{DAS}$.
\end{abstract}

Keywords: sugarcane, straw, weed.

\section{INTRODUÇÃO}

A deposição de palha sobre os solos cultivados implica alterações na qualidade da luz (Ballaré \& Casal, 2000), amplitude térmica
(Hossain et al., 2001), umidade (Hatfield, et al., 2001) e presença de aleloquímicos (Trezzi \& Vidal, 2004) na superficie do solo. Como consequência, a interação entre esses fatores pode contribuir com a supressão

1 Recebido para publicação em 23.10.2012 e aprovado em 26.6.2013.

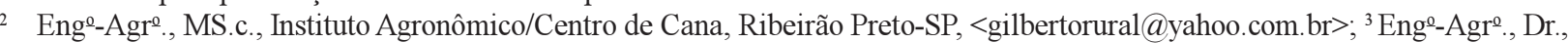
PqC, Inst. Agronômico/Centro de Cana, Rodov. Pref. Antonio Duarte Nogueira, Km 321, 14032-800 Ribeirão Preto-SP, <azania@iac.sp.gov.br>; ${ }^{4}$ Eng-â-Agr ${ }^{\mathrm{a}}$., Dra., PqC, Inst. Agronômico/Centro de Ecofisologia e Biofísica, Avenida Theodureto de Almeida Camargo 1500, Caixa Postal 28, 13001-970 Campinas-SP, <mcdesalvo@hotmail.com>; ${ }^{5}$ Enga-Agr ${ }^{\mathrm{a}}$., Dra., PqC., Inst. Agronômico/Centro de Grãos e Fibras, 13075-630 Campinas-SP, <ebwutke@iac.sp.gov.br>; ${ }^{6}$ Eng ${ }^{\mathrm{a}}-$ Agr $^{\mathrm{a}}$., Doutorando em Agronomia, Fac. Ciênc. Agr. e Vet. Jaboticabal, Via de Acesso Prof. Paulo Donato Castellane s/n, 14884-900 Jaboticabal-SP, <fabricio0_sp@hotmail.com>; 7 Bióloga, Pós-doutoranda, Inst. Agronômico/Centro de Cana, Ribeirão Preto-SP, $<$ andrea.azania@hotmail.com>. 
(Teasdale et al., 1991) ou estímulo à emergência de plantas daninhas.

Nos canaviais colhidos sem a despalha dos colmos pela queima, a deposição da palha evidencia a supressão de plantas daninhas. Em outras situações, a deposição da palha tem estimulado a emergência de Ipomoea spp. e Merremia spp. (Correia \& Kronka, 2010), Euphorbia heterophylla (Monquero et al., 2007) e, mais recentemente, Mucuna aterrima (Campos et al., 2011).

No tocante a $M$. aterrima, pouco é conhecido acerca de sua biologia como planta daninha, pois, segundo Wutke (1993), a espécie é uma leguminosa cultivada para fixar o $\mathrm{N}$ atmosférico ao solo, além de constituir fonte de matéria orgânica pela incorporação de sua massa vegetal. Durante o cultivo da espécie como adubo verde, práticas como a semeadura com sementes dormentes (Wutke et al., 1995) e a incorporação das plantas com sementes próximo à maturação (Nakagawa et al., 2007) são as principais causas de enriquecimento do banco de sementes no solo com os propágulos da espécie.

Posteriormente, entre as culturas instaladas, observam-se fluxos de emergência de $M$. aterrima, sendo a adaptação mais bem observada no agroecossistema da cana-deaçúcar. Seja após plantio ou nas soqueiras da cultura, a massa vegetal formada pela mucuna promove o acamamento, com consequência sobre o acúmulo de sacarose e dificuldade para colheita dos colmos. Mucuna cinerea e Mucuna deeringiana também podem ser utilizadas como adubo verde (Wutke, 1993), porém o potencial de suas plântulas em superar a palha depositada nos canaviais também não foi elucidado. Práticas de manejo mais apropriadas sobre Mucuna spp. podem ser obtidas ao se conhecer a profundidade de emergência, associada ou não a diferentes densidades de palha, bem como o desenvolvimento inicial das plântulas.

Ao observar as elevadas infestações de mucunas nos canaviais e a agressividade no desenvolvimento das plantas, levanta-se a hipótese de que os propágulos do banco de sementes superam diferentes profundidades de solo e, também, camadas de palha. Entretanto, para verificar essa afirmação, este trabalho objetivou estudar a emergência e o desenvolvimento inicial das espécies de Mucuna aterrima, Mucuna cinerea e Mucuna deeringiana sob diferentes profundidades de semeadura e em densidades de palha de canade-açúcar.

\section{MATERIAL E MÉTODOS}

O experimento foi desenvolvido em casa de vegetação durante os meses de outubro a novembro de 2009, utilizando-se como unidades experimentais vasos de plástico com capacidade para 9 L. O delineamento experimental utilizado foi o inteiramente casualizado, com os tratamentos distribuídos em esquema fatorial $3 \times 3 \times 3$, com quatro repetições. $O$ primeiro fator foi constituído por camadas de palha de cana-de-açúcar: $0 \mathrm{~cm}\left(0 \mathrm{tha}^{-1}\right), 5 \mathrm{~cm}\left(9 \mathrm{tha}^{-1}\right)$ e $10 \mathrm{~cm}\left(23 \mathrm{tha}^{-1}\right)$; o segundo fator, por espécies de mucuna (Mucuna aterrima; Mucuna cinerea e Mucuna deeringiana) e o terceiro, pelas profundidades de semeadura (1, 4 e $8 \mathrm{~cm})$.

As sementes das espécies estudadas foram adquiridas de empresa especializada; quanto às espécies de Mucuna aterrima e Mucuna cinerea, devido à dormência natural de suas sementes (Wutke et al., 1995), utilizou-se a metodologia de Trani et al. (1991) para minimizar os efeitos da dormência.

$\mathrm{Na}$ sequência, caracterizou-se o lote de sementes quanto à germinação e viabilidade, segundo preconizado por Brasil (2009): $M$. aterrima apresentou 59 e 98\%; $M$. cinerea, 98 e $100 \%$; e $M$. deeringiana, 57 e $58 \%$, respectivamente.

Na ocasião da semeadura, as sementes foram tratadas com o fungicida dissulfeto de tetrametil-tiran (140 $\mathrm{g} \mathrm{ha}^{-1}$ ) para cada $100 \mathrm{~kg}$ sementes, a fim de evitar contaminação por fungos presentes no solo. A palha do cultivar RB 72454, obtida em área comercial antes da aplicação do herbicida, foi peneirada para retirar as impurezas.

A terra, oriunda de área de pousio, foi peneirada e adubada com $30 \mathrm{~kg} \mathrm{ha}^{-1}$ de $\mathrm{N}$, $120 \mathrm{~kg} \mathrm{ha}{ }^{1}$ de $\mathrm{P}_{2} \mathrm{O}_{5}, 60 \mathrm{~kg} \mathrm{ha}^{-1}$ de $\mathrm{K}_{2} \mathrm{O}$ e $5 \mathrm{~kg} \mathrm{ha}^{-1}$ de $\mathrm{Zn}$, para simular os solos dos canaviais. A coleta teve origem na camada arável de um Latossolo Vermelho Eutroférrico de textura 
argilosa (44\% de argila, 13,2\% de silte e 42,8\% de areia). Como características químicas, apresentou: $\mathrm{pH}$ de 5,$5 ; 27 \mathrm{~g} \mathrm{dm}^{-3}$ de matéria orgânica; $10 \mathrm{mg} \mathrm{dm}^{-3}$ de $\mathrm{P}_{\text {(resina) }} ; 3,5 \mathrm{mmol}_{\mathrm{c}} \mathrm{dm}^{-3} \mathrm{de}$ $\mathrm{K} ; 61 \mathrm{mmol}_{\mathrm{c}} \mathrm{dm}^{-3} \mathrm{de} \mathrm{Ca} ; 16 \mathrm{mmol}_{\mathrm{c}} \mathrm{dm}^{-3} \mathrm{de} \mathrm{Mg}$; e $80 \%$ de V\%.

Na ocasião da instalação do experimento, os vasos tiveram o volume parcialmente preenchido com a terra e foram semeadas dez sementes da espécie almejada. O restante do volume do vaso foi preenchido de modo a constituir a profundidade de semeadura desejada; sobre a superficie da terra foi colocada a densidade de palha. No final, os vasos, com todos os tratamentos de acordo com o delineamento adotado, foram casualizados e colocados sobre bancadas em casa de vegetação. A irrigação foi feita diariamente com $200 \mathrm{~mL}$ de água, que proporcionou condições de umidade suficiente para o pleno desenvolvimento das plantas.

Diariamente, contou-se o número de plantas emergidas, sendo consideradas apenas aquelas que expuseram as folhas cotiledonares até o 20 dia após semeadura (DAS). Os dados dos 10, 15 e 20 DAS foram utilizados para cálculo da porcentagem de emergência (( $\Sigma$ número de plântulas emergidas/ Inúmero de sementes semeadas)*100).

Aos 20 DAS, fez-se também o desbaste das plantas, deixando somente três plantas por parcela; aos 35 DAS, foram avaliadas a altura, a área foliar e a massa seca das plantas. A altura foi obtida medindo-se, em centímetros, as plantas do solo até o meristema apical do ramo principal. A área foliar $(A F)$ das plantas foi obtida pelo integrador de área, modelo Li-3100 (Licor Inc., Lincoln-EUA). Para obtenção da massa seca, as plantas foram cortadas rente ao solo, acondicionadas em sacos de papel e, posteriormente, levadas para estufa de circulação forçada de ar à temperatura de $70{ }^{\circ} \mathrm{C}$, até peso constante.

As variáveis avaliadas foram submetidas à análise de variância pelo teste $\mathrm{F}$, e as médias, comparadas pelo teste de Tukey a $5 \%$, utilizando-se o programa estatístico computacional ESTAT. Os dados de porcentagem de emergência das plântulas foram transformados em arc sen (raiz $(x+0,5) / 100)$.

\section{RESULTADOS E DISCUSSÃO}

M. aterrima apresentou valores de emergências similares em qualquer profundidade de semeadura ou densidade de palha aos 10 DAS. Esses resultados sugerem que a espécie é indiferente à presença ou ausência de palha e supera até $8 \mathrm{~cm}$ de solo e mais $10 \mathrm{~cm}$ de palha, com facilidade para expor as partes aéreas à superficie (Tabela 1).

Ainda aos 10 DAS, resultados similares foram observados para a emergência das plântulas de $M$. deeringiana. As interações entre densidades de palha e espécies (A x B) e espécies e profundidade de semeadura (B x C) demonstraram menor emergência das plântulas nos tratamentos com $23 \mathrm{t} \mathrm{ha}^{-1}$ de palha e com $8 \mathrm{~cm}$ de profundidade de semeadura, respectivamente (Tabela 1 ).

Os resultados das interações mostraram que $M$. cinerea apresentou emergência de $99,17 \%$ quando na ausência de palha e de $70,83 \%$ na densidade de palha de $23 \mathrm{t} \mathrm{ha}^{-1}$; a espécie também apresentou $94,17 \%$ quando

Tabela 1 - Desdobramento da interação A x B (palha x espécies) e C x B (profundidade de semeadura x espécies) para porcentagem de emergência ${ }^{1 /}$ aos 10 dias após semeadura de Mucuna spp. Campinas-SP

\begin{tabular}{|c|c|c|c|c|}
\hline \multirow{5}{*}{ 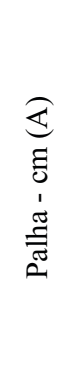 } & & \multicolumn{3}{|c|}{ Espécie (B) } \\
\hline & & MA & $\mathrm{MC}$ & MD \\
\hline & $\begin{array}{c}10 \\
\left(23 \mathrm{t} \mathrm{ha}^{-1}\right)\end{array}$ & $\begin{array}{c}38,55 \mathrm{Ab} \\
(39,17)\end{array}$ & $\begin{array}{c}60,85 \mathrm{Ba} \\
(70,83)\end{array}$ & $\begin{array}{c}33,72 \mathrm{Bb} \\
(32,50)\end{array}$ \\
\hline & $\begin{array}{c}5 \\
\left(9 \mathrm{tha}^{-1}\right)\end{array}$ & $\begin{array}{c}39,77 \mathrm{Ac} \\
(40,83)\end{array}$ & $\begin{array}{c}82,52 \mathrm{Aa} \\
(95,83)\end{array}$ & $\begin{array}{c}51,96 \mathrm{Ab} \\
(60,83)\end{array}$ \\
\hline & $\begin{array}{c}0 \\
\left(0 \mathrm{tha}^{-1}\right)\end{array}$ & $\begin{array}{c}43,78 \mathrm{Ab} \\
(47,50)\end{array}$ & $\begin{array}{c}88,50 \mathrm{Aa} \\
(99,17)\end{array}$ & $\begin{array}{c}50,75 \mathrm{Ab} \\
(59,17)\end{array}$ \\
\hline$\because 0$ & 1 & $\begin{array}{c}39,79 \mathrm{Ac} \\
(40,83)\end{array}$ & $\begin{array}{c}81,14 \mathrm{Aa} \\
(94,17)\end{array}$ & $\begin{array}{c}53,92 \mathrm{Ab} \\
(64,17)\end{array}$ \\
\hline 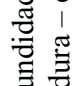 & 4 & $\begin{array}{c}41,56 \mathrm{Ab} \\
(44,17)\end{array}$ & $\begin{array}{c}80,08 \mathrm{Aa} \\
(92,50)\end{array}$ & $\begin{array}{c}42,23 \mathrm{Bb} \\
(45,00)\end{array}$ \\
\hline 을 & 8 & $\begin{array}{c}40,75 \mathrm{Ab} \\
(42,50)\end{array}$ & $\begin{array}{c}70,64 \mathrm{Ba} \\
(79,17)\end{array}$ & $\begin{array}{c}40,28 \mathrm{Bb} \\
(43,33)\end{array}$ \\
\hline
\end{tabular}

Mucuna aterrima (MA), Mucuna cinerea (MC), Mucuna deeringiana (MD); letra maiúscula é comparada nas colunas e letra minúscula, na linha; Dias após semeadura (DAS); dados originais entre parênteses; 1 dados transformados em $\operatorname{arcsen}(\operatorname{raiz}(x+0,5) / 100)$.

Planta Daninha, Viçosa-MG, v. 31, n. 2, p. 313-317, 2013 
semeada a $1 \mathrm{~cm}$ de profundidade e $79,17 \%$ na profundidade de $8 \mathrm{~cm}$ (Tabela 1). Assim, constata-se que, mesmo sendo a espécie com o maior percentual de emergência, ainda é suscetivel aos efeitos da palha e de profundidade de semeadura.

$\mathrm{Na}$ literatura, verificou-se que sementes de plantas daninhas com tamanho inferior ao das espécies estudadas - a exemplo de Digitaria sanguinalis e Portulaca oleraceae também conseguiram entrar no processo de germinação e expor as partes aéreas à superfície quando posicionadas até $3,6 \mathrm{~cm}$ de profundidade; contudo, com $10 \mathrm{~cm}$ de solo, $85 \%$ das sementes não germinaram. Essas sementes entraram em dormência e passaram a contribuir com o aumento do banco de sementes no solo (Benvenuti et al., 2001).

Para as sementes entrarem no processo de dormência ou germinação, existem diversos fatores intrínsecos e extrínsecos envolvidos. $\mathrm{O}$ efeito físico da cobertura vegetal (Teasdale et al., 1991), a qualidade da luz incidente no solo (Ballaré \& Casal, 2000), as oscilações de temperatura (Egley \& Duke, 1985), as faixas de pH e o potencial osmótico do solo (Susko \& Hussein, 2008) são alguns dos fatores extrinsecos que mais influenciam as sementes nos processos de dormência ou germinação. A dureza do tegumento (Wutke et al., 1995) e a relação entre o tamanho das sementes (Giomo et al., 2008) e a reserva do embrião (Chauhan \& Johnson, 2008) são alguns dos fatores intrinsecos que influenciam também os processos de germinação e dormência.

Ao observar o estabelecimento inicial das plântulas, pode-se ressaltar que o efeito fisico da palha ( $23 \mathrm{t} \mathrm{ha}^{-1}$ ) e a profundidade de semeadura de até $8 \mathrm{~cm}$ minimizaram a emergência das espécies até os 10 DAS, porém não o suficiente para a total supressão (Tabela 1); aos 15 e 20 DAS, não se observou mais efeito supressor sobre as espécies (dados não mostrados). Esses resultados sugerem ao produtor a intervenção com manejos na pré-emergência ou na pós-emergência inicial dessas espécies.

A altura das plantas de mucuna, avaliada aos 35 DAS, não foi influenciada pelas espessuras da camada de palha ou pelas profundidades de semeadura (Tabela 2).
Mucuna deeringiana apresentou a menor altura; entretanto, essa espécie é conhecida como anã e tem hábito de crescimento determinado, com ciclo curto e menor produção de massa vegetal em relação às demais espécies de Mucuna spp. (Calegari et al., 1992).

Quanto à área foliar, os tratamentos com 23 e $9 \mathrm{t} \mathrm{ha}^{-1}$ de palha proporcionaram os maiores valores para essa variável, diferenciando significativamente do tratamento sem palha (Tabela 2). M. aterrima e $M$. cinerea comportaram-se de maneira similar para área foliar, diferenciando de $M$. deeringiana, provavelmente devido ao hábito de crescimento determinado e às características específicas da espécie.

Tabela 2 - Média para altura (cm), número de trifólios, área foliar $\left(\mathrm{cm}^{2}\right)$ e índice de área foliar por planta, proporcionados pelas diferentes profundidades de semeadura e camada de palha de cana-de-açúcar sobre as espécies de Mucuna aos 35 DAS. Campinas-SP

\begin{tabular}{|c|c|c|c|}
\hline Causa de variação & $\begin{array}{c}\text { Altura } \\
(\mathrm{cm})\end{array}$ & $\begin{array}{c}\text { Área foliar } \\
\left(\mathrm{cm}^{-2}\right)\end{array}$ & $\begin{array}{c}\text { Massa seca } \\
(\mathrm{g})\end{array}$ \\
\hline \multicolumn{4}{|l|}{ Espessuras palha - cm (A) } \\
\hline $10\left(23 \mathrm{tha}^{-1}\right)$ & $113,45 \mathrm{a}$ & $820,76 \mathrm{a}$ & $2,66 \mathrm{a}$ \\
\hline $5\left(9 \mathrm{t} \mathrm{ha}^{-1}\right)$ & $115,86 \mathrm{a}$ & $831,72 \mathrm{a}$ & $2,74 \mathrm{a}$ \\
\hline $0\left(0 \mathrm{t} \mathrm{ha}^{-1}\right)$ & $112,73 \mathrm{a}$ & $717,42 \mathrm{~b}$ & $2,29 \mathrm{~b}$ \\
\hline \multicolumn{4}{|l|}{ Espécies (B) } \\
\hline Mucuna aterrima & $123,95 \mathrm{a}$ & $839,23 \mathrm{a}$ & $2,76 \mathrm{a}$ \\
\hline Mucuna cinerea & 121,79 a & $807,58 \mathrm{a}$ & $2,84 \mathrm{a}$ \\
\hline Mucuna deeringiana & $96,29 \mathrm{~b}$ & $723,09 \mathrm{~b}$ & $2,09 \mathrm{~b}$ \\
\hline \multicolumn{4}{|l|}{$\begin{array}{l}\text { Profundidades de } \\
\text { semeadura }-\mathrm{cm}(\mathrm{C})\end{array}$} \\
\hline 1 & $112,38 \mathrm{a}$ & $788,66 \mathrm{a}$ & $2,60 \mathrm{a}$ \\
\hline 4 & $113,70 \mathrm{a}$ & $783,94 \mathrm{a}$ & $2,53 \mathrm{a}$ \\
\hline 8 & $115,97 \mathrm{a}$ & $797,29 \mathrm{a}$ & $2,56 \mathrm{a}$ \\
\hline $\mathrm{F}(\mathrm{A})$ & $0,51 \mathrm{~ns}$ & $11,73 * *$ & $15,59 * *$ \\
\hline $\mathrm{F}(\mathrm{B})$ & $44,68^{* *}$ & $10,63^{* *}$ & $47,74 * *$ \\
\hline $\mathrm{F}(\mathrm{C})$ & $0,62 \mathrm{~ns}$ & $0,13 \mathrm{~ns}$ & $0,37 \mathrm{~ns}$ \\
\hline $\mathrm{F}(\mathrm{AxB})$ & $0,34 \mathrm{~ns}$ & $0,53 \mathrm{~ns}$ & $0,59 \mathrm{~ns}$ \\
\hline $\mathrm{F}(\mathrm{A} \times \mathrm{C})$ & $2,02 \mathrm{~ns}$ & $0,32 \mathrm{~ns}$ & $0,58 \mathrm{~ns}$ \\
\hline $\mathrm{F}(\mathrm{B} \times \mathrm{C})$ & $0,63 \mathrm{~ns}$ & $0,59 \mathrm{~ns}$ & $0,44 \mathrm{~ns}$ \\
\hline $\mathrm{F}(\mathrm{A} \times \mathrm{B} \times \mathrm{C})$ & $0,95 \mathrm{~ns}$ & $0,52 \mathrm{~ns}$ & $0,71 \mathrm{~ns}$ \\
\hline Dms & 7,78 & 62,22 & 0,20 \\
\hline $\mathrm{CV}(\%)$ & 12,11 & 13,98 & 13,98 \\
\hline
\end{tabular}

Dias após semeadura (DAS); índice de área foliar (IAF); ns - não significativo; $* *$ - significativo a $1 \%$ de probabilidade pelo teste $\mathrm{F}$; $\mathrm{CV}$ - coeficiente de variação; dms - diferença mínima significativa. 
Aos 35 DAS, observou-se menor massa seca no tratamento sem palha, enquanto os tratamentos com 9 e $23 \mathrm{t} \mathrm{ha}^{-1}$ foram iguais estatisticamente. Esse efeito foi positivo devido à manutenção de água no substrato; assim, essas plantas puderam manter seus estômatos abertos, com consequente maior assimilação de carbono pela fotossintese, produzindo maior quantidade de carboidratos.

De modo geral, verificou-se que todas as espécies de Mucuna spp. apresentaram maior emergência quando as sementes se encontravam próximas à superficie do solo e sem a presença de palha. Assim, a hipótese de que os propágulos do banco de sementes superam diferentes profundidades de solo e camadas de palha confirma-se por este trabalho, ao passo que Mucuna aterrima, Mucuna cinerea e Mucuna deeringiana superam até $23 \mathrm{tha}^{-1}$ de palha e até $8 \mathrm{~cm}$ de profundidade de semeadura, expondo as partes aéreas à superficie a partir dos 10 DAS, sendo capazes também de desenvolver-se em altura $(>96 \mathrm{~cm})$, área foliar $\left(>723 \mathrm{~cm}^{-2}\right)$ e acúmulo de massa seca (> 2,09 g por planta) até os 35 DAS.

\section{LITERATURA CITADA}

BALLARÉ, C. L.; CASAL, J. J. Light signals perceived by crop and weed plants. Field Crops Res., v. 67, n. 2, p. 149-160, 2000.

BENVENUTI, S.; MACCHIA, M.; MIELE, S. Quantitative analysis of emergence of seedlings from buried weed seeds with increasing soil depth. Weed Sci., v. 49, n. 4, p. 528-535, 2001.

BRASIL. Ministério da Agricultura, Pecuária e Abastecimento. Regras para análise de sementes. Brasília: Ministério da Agricultura, Pecuária e Abastecimento. Secretaria de Defesa Agropecuária - Mapa/ACS, 2009. 399 p.

CALEGARI, A. et al. Caracterização das principais espécies de adubo verde. In: COSTA, M. B. B. (Coord.). Adubação verde no sul do Brasil. Rio de Janeiro: AS-PTA, 1992. p. 207-327.

CAMPOS, L. H. F. et al. Emergência de Merremia cissoides, Mucuna aterrima e Neonotonia wightii sob diferentes profundidades de semeadura e quantidades de palha de cana-deaçúcar. Planta Daninha, v. 29, p. 975-980, 2011. (Número Especial)

CHAUHAN, B. S.; JOHNSON, D. E. Seed germination and seedling emergence of nalta jute (Corchorus olitorius) and redweed (Melochia concatenata): Important broadleaf weeds of the tropics. Weed Sci., v. 56, n. 6, p. 814-819, 2008.
CORREIA, N. M.; KRONKA JR., B. Controle químico de plantas dos gêneros Ipomoea e Merremia em cana-soca. Planta Daninha, v. 28, p. 1143-1152, 2010. (Número Especial)

EGLEY, G. H.; DUKE, S. Physiology of weed seed dormancy and germination. In: DUKE, S. O. Weed physiology. Ireproduction and ecophysiology. Florida: CRC Press, 1985. p. 27-64.

GIOMO, G. S.; NAKAGAWA, J.; GALLO, P. B. Beneficiamento de sementes de café e efeitos na qualidade fisiológica. Bragantia, v. 67, n. 4, p. 1011-1020, 2008.

HATFIELD, J. L.; SAUER, T. J.; PRUEGER, J. T. Managing soils to achieve greater water use efficiency: a review. Agron. J., v. 93, n. 3, p. 271-280, 2001.

HOSSAIN, M. A. et al. Influence of temperature levels and planting time on the sprouting of rhizome-bud and biomass production of torpedograss (Panicum repens L.) in Okinawa island, southern Japan. Weed Biol. Manag., v. 1, n. 3, p. 164-169, 2001.

MONQUERO, P. A. et al. Eficácia de herbicidas em diferentes quantidades de palha de cana-de-açúcar no controle de Euphorbia heterophylla. Planta Daninha, v. 25, n. 3, p.613-619, 2007.

NAKAGAWA, J. et al. Intensidade de dormência durante a maturação de sementes de mucuna-preta. R. Bras. Sementes, v. 29, n. 1, p. $165-170,2007$.

SUSKO, D. J.; HUSSEIN, Y. Factors affecting germination and emergence of Dame's Rocket (Hesperis matronalis).

Weed Sci., v. 56, n. 3, p. 389-393, 2008.

TEASDALE, J. R.; BESTE, C. E.; POTTS, W. E. Response of weeds to tillage and cover crop residue. Weed Sci., v. 39, n.2, p. 195-199, 1991.

TRANI, P. E.; BULISANI, E. A.; BRAGA, N. R. Adubação verde. $2^{\mathrm{a}}$ impressão. Campinas: Coordenadoria de Assistência Técnica Integral - CATI, 1991. 13 p. (Boletim Técnico, 197).

TREZZI, M. M.; VIDAL, R. A. Potencial de utilização de cobertura vegetal de sorgo e milheto na supressão de plantas daninhas em condição de campo: II - Efeitos da cobertura morta. Planta Daninha, v. 22, n. 1, p. 1-10, 2004.

WUTKE, E. B.; MAEDA, J. A.; PIO, R. M. Superação da dormência de sementes de mucuna-preta pela utilização de "calor seco". Sci. Agric., v. 52, n. 3, p. 482-490, 1995.

WUTKE, E. B. Adubação verde: manejo da fitomassa e espécies utilizadas no Estado de São Paulo. In: WUTKE, E. B.; BULISANI, E. A.; MASCARENHAS, H. A. A. (Eds.). Curso sobre adubação verde no Instituto Agronômico. Campinas: Instituto Agronômico de Campinas, 1993. p. 17-29. (Documentos IAC, 35) 